Vol. 3, No. 1, 2021

https://doi.org/10.23939/cds2021.01.021

UDK 53 (075.8);519.21;621.417

P. Kosobutskyi , M. Lobur, Yu. Matviiv-Lozynska

Lviv Polytechynic National University

\title{
METHOD OF STATISTICAL IMITATION, ITS CREATOR S. ULAM AND BASIC PRINCIPLES OF APPLICATION FOR RANDOM PROCESSES MODELING
}

(C) Kosobutskyi P., Lobur M., Matviiv-Lozynska Yu., 2021

Abstract: The article is dedicated to famous Lviv residents, the author of the Monte Carlo method, and mathematician Marco Katsu. The method of Monte Carlo methodical algorithms for modeling statistical regularities of light passage through optically inhomogeneous media is briefly described in the article.

Keywords: Monte Carlo method, light scattering by turbid media

\section{Introduction}

1. Lviv mathematician Stanislaw Ulam is a creator of statistical modeling method or Monte Carlo method. Famous mathematician Stanislaw Ulam was born in Lviv on 13 April 1909. His father, Jozef Ulam, was born in Lviv and was a lawyer, and his mother, Anna, was born in Stryi. During 1919-1927 he was studying in Lviv Gymnasium, and during 1927-1933 in technical faculty of Lviv Polytechnic Institute. During his study in Lviv Polytechnic Institute, Stanislaw Ulam attended a course of theoretical physics by famous Polish theoretical physicist Wojciech Rubinowicz, who was German physicist Sommerfeld's student. S.Ulam took active participation in scientific seminars of Lviv school of mathematics that were held in Rome and Scottish Cafe with the involvement of Stefan Banach, Waclaw Sierpinski and Stanislaw Mazur.

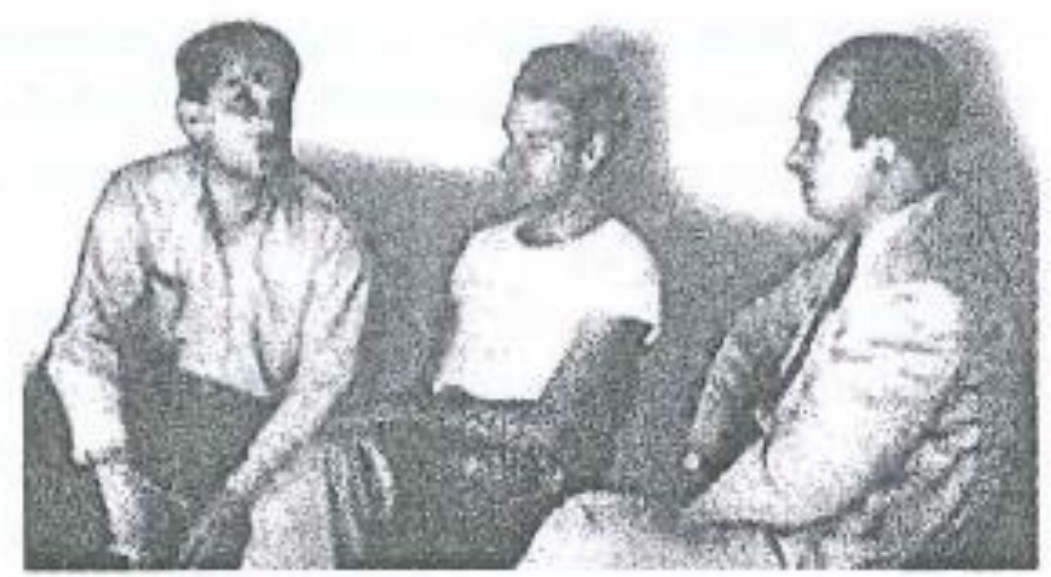

Fig. 1. S.Ulam (on the left) with famed physicist Richard Feynman (in the middle) and mathematician von Neumann (on the right) 
As he recalled it, around 1929-1930 at the time of mathematical discussions in coffee houses young at that time mathematician Stanislaw Mazur raised a question about abstract machines, which can copy themselves. These ideas preceded theory of abstract machines, which would be developed by famous Hungarian mathematician, participant of Manhattan Project John von Neumann. John von Neumann came to Lviv for the first time for the congress of mathematicians in 1927. Unfortunately, S.Ulam couldn't meet him because of his final examination. Lviv department of Polish Mathematical Society suggested to student Ulam to hold the post of secretary. It gave him the opportunity to take part in international mathematical congresses and to expand the circle of contacts with leading mathematicians.

In 1932 S.Ulam was invited to make a report on International mathematical congress, which occured in Zurich. In a short while, from 1934 S.Ulam began to correspond with famous scientist von Neumann and discussed with him relevant problems of mathematics. John von Neumann emigrated to USA in 1930 and worked in Institute for Advanced Study (Prinston). Exactly at Ulam's invitation in 1937 von Neumann back again to Lviv for being engaged in scientific seminar. John von Neumann recommended S.Ulam to emigrate to the USA. Then Ulam joined a group of mathematicians, which was lead by von Neumann himself. This group began intensive mathematical investigation within Manhattan Project in 1943. In 1945 von Neumann mathematically coherent the use of an explosive method of detonation of an atomic bomb and suggested principle of building electronic computer, which lately would be named in his honor of EDVAC. S.Ulam with von Neumann in 1949 offered decisive for Manhattan Project implementation method of statistical testing of complex systems with different implementation possibilities, which was later called Monte Carlo method.

The main difference between Monte Carlo method and researches, which were conducted in the field of statistical modeling, is that Monte Carlo modelling overturned the standard view of how problem must be solved, using the methods of probability theory and mathematical statistics. Previously it was assumed that fistly you need to study the deterministic problem and then to use the simulation. In Monte Carlo modelling is allowed that one must have a deterministic problem and find its stochastic counterpart. It is the general principle that isn't based on specific physical ideas and can be used for solving tasks in physics, chemistry, biology and economic etc. S.Ulam with Edward Teller put forward an idea of hydrogen bomb in 1950, which was later (1954) theoretically coherent by O. Lavrentiev and A.Sakharov. Afterwards, in 1955, he put forward an idea of nuclear pulse propulsion for an interplanetary space Project Orion. Stanislaw Ulam worked closely with prominent physicist, a Nobel Prize winner for physics (1965), Richard Feynman. In the photo Stanislaw Ulam with another Lviv mathematician Mark Kac, student of Hugo Steinhaus, professor of mathematics at The Rockefeller University, with which he co-authored the book. As Ulam remembered, editors of British Encyclopaedia proposed him and Mark Kac to write a mathematics article for the special supplement "Britanica". This article was also published as separate collective writing, which name was "Mathematic and Logic". Lately it was translated into French, Spanish, Russian, Czech and Japan languages.
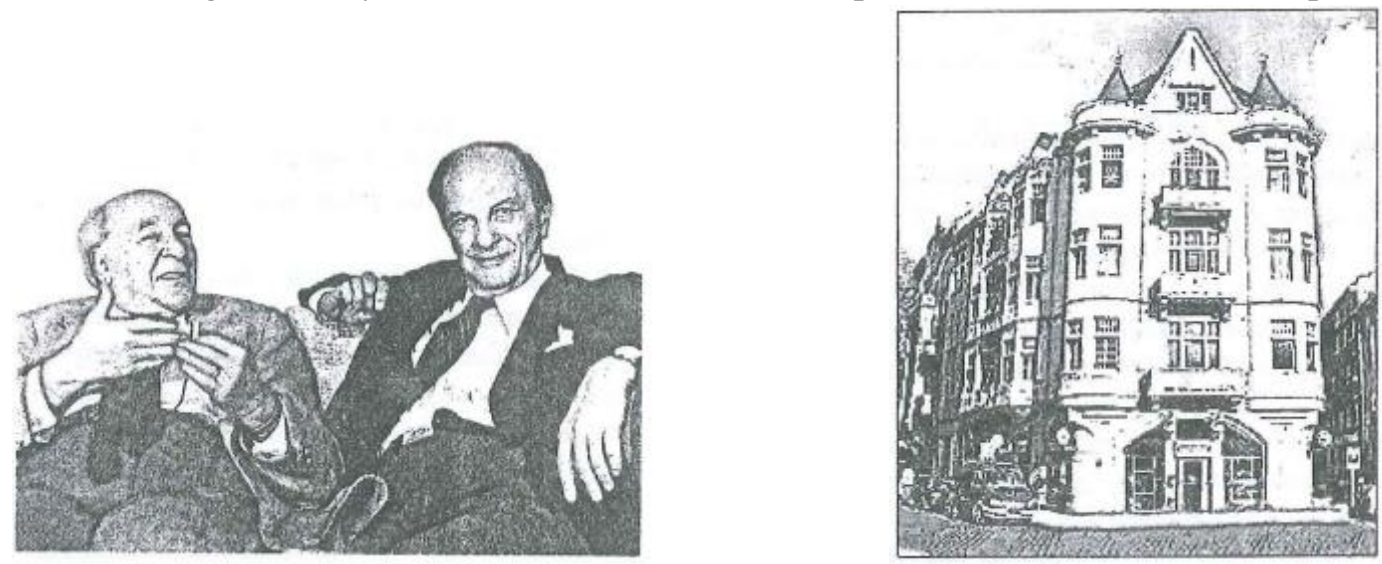

Fig. 2. Mark Kac (1914-1984) and Scottish Coffee Houses took place on the Stanislaw Ulam (19091984) ground floor of this house. Next door (on the right, 1 Saksahanskyi str.). Now there is editorial staff of "World of physics" magazine 
S. Ulam remembered with warmth years spent in Lviv. He found it difficult to perceive death of well- known Lviv mathematician Stefan Banach after war. After Second World War S.Ulam renovated relationships with Polish mathematicians. They gave to him famous Stefan Banach's workbook with manuscripts of scientific seminars (from the words of Steinhaus, workbook was saved and given by Stefan Banach's son - neurosurgeon), which was translated by S.Ulam into English and prepared to publishing named "The Scottish Book" [The Scottish Book: A Collection of problems. Translated from a notebook kept at the Scottish Coffee House for the use of the Lwowe Section. Polish Mathematical Society Los Alamos, N. Mex: Los Alamos Scientific Laboratory, 1957]. S.Ulam published a monograph "Unsolved Problems of Mathematics" in 1960. Stanislaw Ulam died in Santa Fe (New Mexico, USA) on 13 May 1984, was burnt in Paris at the Montmartre Cemetery.

The purpose of this message is to attract attention to unfairly forgotten Lviv mathematician Stanislaw Ulam. However, it is difficult to succinctly display all facets of his scientific achievements and make it better than authors of writing [1] to mark the scientist's 100th birthday. Mykhailo Zarichyi, professor of Ivan Franko National University of Lviv aptly noted a tribute of great respect and recognition of merit of Lviv school of mathematics of Steinhaus-Banach-Ulam-Sierpinski-Mazur. He read in one of the scientific journals that between two worldwide wars there were three European capitals of mathematics: Paris, Goettingen and Lviv. So we hope that Lviv community will support the idea to reconstruct historical places of great works of mathematics at Rome and Scottish Coffee Houses.

2. Method of Ulam or the Monte Carlo method (MCM). By idea of Monte Carlo method we evaluate the value of a defined integral $\int_{a}^{b} f(x) d x$ at the interval $[\mathrm{a}, \mathrm{b}]$ means to evaluate the value of $h(b-a) \frac{n_{k}}{n}$. Where $h(b-a)$ is the area of the rectangle, inside which lies the graph of the function $f(x)$ $; \frac{n_{k}}{n}$ is the ratio between random points under the curve of $f(x)$ and to the total number $\mathrm{n}$ of randomly generated pairs which coordinates are $x_{i}\left(a \leq x_{i} \leq b\right)$ and $y_{i}\left(a \leq y_{i} \leq b\right)$ with uniform distribution. Therefore, modeling random numbers with a given distribution law is an important component of the MCM

3. Modeling the scattering of photons in turbid media [2 - 4] using MCM. When physical and technical process are modeled, often a probability distribution is shown/depicted through an ensemble. Instead of dealing with one variable and its probability distribution, consider any large set of values taken by the number of $n$ variables in given region. Moreover a number of variables, whose values fall within interval from $x$ to $x+d x$, is equal to $n P(x) d x$. Then a probability distribution is replaced with a probability density of a large number of samples, that has no influence on a final result, but is convenient method for illustrative description of randomness. So, the probability that $x$ belongs to the certain element of a phase space $d x$, is equal to the phase of the sample states of an ensemble within this interval.

Substitution of an ensemble for real physical systems leads to that every physical quantity $Y(t)$ becomes a stochastic process, means value and moments of which can connect with observations. Furthermore, there is the time and ensemble averaging. So, thanks to the theory of Brownian motion it is known that the average velocity is depicted by the macroscopic damping law, while the autocorrelation function determines a diffusion coefficient. A physical system actually often consists of a number of identical particles, which to a certain extent form physical realization of an ensemble. For instance, molecules of ideal gas form an ensemble that represent the Maxwell-Boltzmann velocity distribution function. A distribution of electrons scattered from a target is a probability distribution by angles of deviation. MMC is used to solve two types of problems: process modelling with impact of stochastic process as well as modeling of complex multidimensional physical objects, not related to some randomness, but allows an artificial probabilistic interpretation. In both cases essence of the method lies in finding possible 
values of a random variable, that adequately represents the process, and estimation mean values from numerous implementation of process that is modeled.

Modelling of the first type of problems. This type of the problems includes transportation and queueing theory problems, calculation of product quality and reliability, a behavior in a group of particles etc. If theoretical methods allow to calculate precisely intersection of interaction of an elementary particles with scattering center or particular atom of matter then modeling by the method of play of random behavior with microparamaters for individual particles such as velocity, free path or the scattering angle. Based on parameters a collection of characteristics of the investigated process can be estimated.

Stochastic processes, as scattering of photons in randomly heterogeneous medium have influence on processes that are modeled in problems of the first type. There is applied so-called method of trajectory integration, which can be divided into analytical, if corresponding problem is solved by approximate analytical method and stochastic, as in the case of light propagation task in randomly heterogeneous medium. Метод of distribution of Browning particles and in the flows of particles is one of analytical methods, which uses certain approximation for problem solving. MCM is a stochastic method for modeling of the scattering in randomly heterogeneous medium.

4. Modelling of the photons scattered in a tissue . It is known [5-9] that the attenuation regularities of photon flux (transport) in optically inhomogeneous medium with random fluctuations of interaction centers are usually modeled using statistical Monte Carlo. This is a flexible, but accurate approach to simulate the process in which local rules of photon migration represented in the form of probability distribution of random variables, such as the displacement step size between two photons scattering acts, azimuthal and polar deviation angles of the direction of this step.

MCM is equivalent to the modeling of the photon migration by analyzing the differential equations of the radiation transport. However, its analytical solutions are often impossible to obtain, while Monte Carlo allows achieving the solution of the problem with any precision by increasing the number of photons. Through the use the laser light sources, this approach has got a wide practical application especially in biomedicine [7-8]. MCM in scattering medium optics is the technique of obtainment and statistical analysis of the trajectories of a large number of scattered photons. In essence, it is a set of procedures for constructing ensembles of random numbers and functions, statistical moments of which are been determined. As the statistical characteristic of the light field in optics of randomly inhomogeneous medium can be defined as the ensembles of photon trajectories and random waves, the approach based on the idea of the radiation propagation as a photons flux is called the "corpuscular" and approach based on the wave conception "wave".

On other hand, the probabilistic laws on the interaction of elementary particles (photons,neutrons, electrons etc) with matter are known. Mainly there are the parameters determined of these procesess, related to presence of many particles, when their statistical regularities,such as flow, density, etc are topical. Consider photons (quanta of the electromagnetic radiation) with energy $h v$ passing through a plane-parallel medium with the thickness $h$. For simplification take into account that angle of entry of a photon in a medium is equal to zero. In the medium photons are scattered and absorbed (Fig. 3,a). In the process «1»a photon after elastic scattering comes out of medium opposite side; in the process « 2 » a photon after elastic scattering comes back to previous medium, and the process « $2 »$ it is absorbed. Each of considered processes occurs with certain probability, whose values must be calculated.

Modeling of a photon trajectory. Based on linear reduction of elementary light intensity $d I$, elementary thickess of the layer $d h: d I=-\mu d h$ is grounded that mean free path of a photon $\lambda$, as the distance between two sequential processes of interaction of photons through scattering with the probability $p_{S}=\frac{\mu_{S}}{\mu}$ and absorption with the probability $p_{\alpha}=\frac{\mu_{\alpha}}{\mu}$, де $\mu=\mu_{S}+\mu_{\alpha}$, if $\mu=$ const $>0$, then probability density function of an exponential distribution with the parameter $\mu$ : $p(\lambda)=\mu e^{-\mu \lambda}, \tau(\lambda)=\mu \lambda$ and the cumulative distribution function $F(\lambda)=1-e^{-\mu \lambda}, \quad \lambda>0$ are correct. 
Therefore, under these conditions a random variable $\lambda$ may be modeled by the formula $\lambda=-\frac{1}{\mu} \ln r$, where $r \in[0 ; 1]$. The meaning of free path is equal to $\langle\lambda\rangle=\frac{1}{\mu}$, and the variance $-\frac{1}{\mu^{2}}$.Vector position is specified with spherical coordinates $\theta, \phi$, which are related to cartesian coordinates as shown in Fig. 3,b. So, when a fallen photon on a plane parallel plate is directed along the $Z$ axis, then $\left(\begin{array}{l}k_{X} \\ k_{Y} \\ k_{Z}\end{array}\right)=\left(\begin{array}{l}0 \\ 0 \\ 1\end{array}\right)$ and correlation for computation scattered photon coordinates are as follows: $x=x_{0}-\frac{\ln r}{\mu_{S}+\mu_{\alpha}} \sin \theta \cos \phi$, $y=y_{0}-\frac{\ln r}{\mu_{S}+\mu_{\alpha}} \sin \theta \sin \phi, \quad z=z_{0}-\frac{\ln r}{\mu_{S}+\mu_{\alpha}} \cos \theta$.
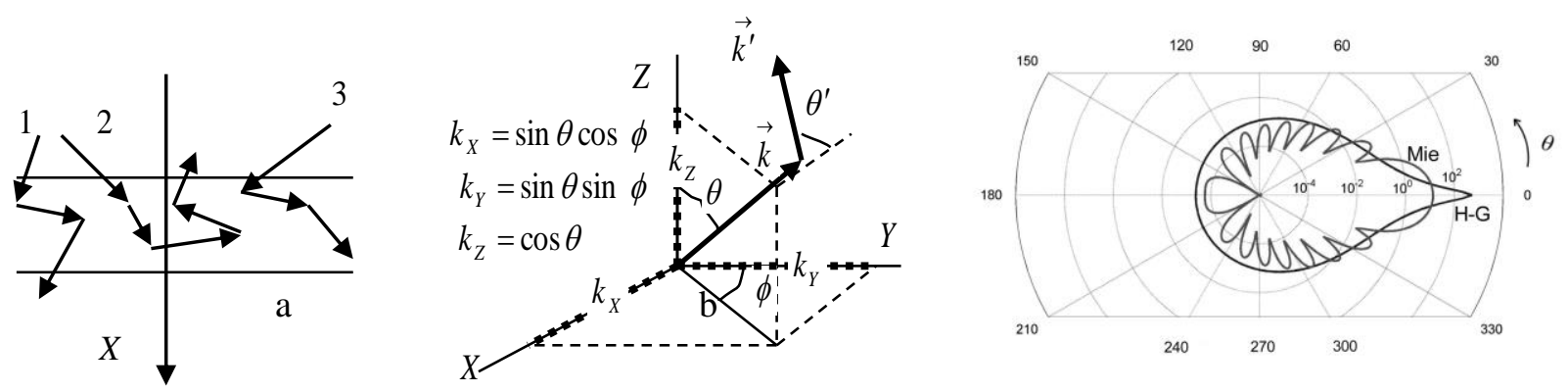

Fig. 3

When the fallen photon moves along $(0,0,1)$, then conversion of coordinates of random scattering center to local coordinates $(x, y, z) \rightarrow\left(x^{\prime}, y^{\prime}, z^{\prime}\right)$ means to rotate the coordinate axes $(x, y, z)$ about the $z$ axis through the angle of $\phi$ and the further rotation of intermediate coordinate system $\left(x^{\prime \prime}, y^{\prime \prime}, z^{\prime \prime}\right)$ about the $y^{\prime \prime}$ axis through the angle $\theta$. The cosine of the zenith angle $\cos \theta$ is within unit interval $[-1 ;+1]$, and the azimuthal angle $\phi$ is within interval $[0 ; 2 \pi]$. The probability distribution of the azimuthal angle $r=\int_{0}^{\phi} \frac{1}{2 \pi} d \phi=\frac{\phi}{2 \pi}$, where $\phi=2 \pi r(r \in[0 ; 1])$. The function for modeling of the zenith angle depends on value of anisotropy parameter $g$. If the scattering is isotropic $g=0$, then $\cos \theta=2 r-1$. It's so-called a transport approximation.

Henyey-Greenstein indicatrix and a phase function. In figure 1,c is shown indicatrix of scattering for different values $g$. For their describtion Henyey and Greenstein introduced the function $p(\cos \theta)=\frac{1-g^{2}}{\left(1+g^{2}-2 g \cos \theta\right)^{3 / 2}} \quad(g<1)$. The function $p(\cos \theta)$ which characterizes the diagram of photon scattering on a individual particle, is called phase function. The function defines the probability that the value of a scattering angle fall within the interval $[\theta ; \theta+d \theta]$ and equals to $2 \pi p(\cos \theta) \sin \theta d \theta$ as well as meets the condition of normalisation $\frac{1}{4 \pi} \int_{4 \pi} p(\cos \theta) d \Omega=1$ in the case of the transparent medium or 
$\frac{1}{4 \pi} \int_{4 \pi} p(\cos \theta) d \Omega\left\langle 1 \quad\right.$ absorbed $\left(\frac{1}{4 \pi} \int_{4 \pi} p(\cos \theta) d \Omega=\frac{\mu_{S}}{\mu_{\alpha}+\mu_{S}}\right)$, if centers are distributed chaotically . For $g=0$ the phase function $p(\cos \theta)$ is not dependent on the angle $\theta$.

If $g=1$, then this is Mie's forward scattering. In the case $g=0$, it is isotropic in all directions of the Rayleigh type. In polar coordinates anisotropy parameter is

$$
g=\frac{\int_{4 \pi} p(\cos \theta) \cos \theta d \Omega}{\int_{4 \pi} p(\cos \theta) d \Omega}=
$$

$2 \pi \int_{0}^{\pi} p(\cos \theta) \cos \theta \sin \theta d \theta$, where $g=\langle\cos \theta>$. Since a direction of photon scattering is random, then $\int_{0}^{\theta} p(\cos \theta) d \theta=r$ and 0 . For most biological tissue $0.7<g<0.99$, in other words $8^{0}<\theta<45^{0}$. Biological objects are highly scattering media, where process of light transmition has a diffuse character.

Weight characteristics. After Metropolis' and Ulam's papers had been published, investigation of possible optimization on MCM was started in 1950th. It was noticed that a lot of the computation time was spent on estimation of trajectory of scattered particles, which contribution to a final result may be not topical enough . For this purpose weight method of optimization was developed, where virtual scattered particles are given so-called weight $-\mathrm{a}$ factor that takes the largest value in direction of the space where its scattering is the most probable. A weight of a particle changes due to absorption as well. Sometimes intermediate steps are used - splitting of a played point with a random coordinate $x$ to several with coordinates $\left(x_{i}, y_{j}, j=1, \ldots i\right)$, that is, the scattering of the played photon is given as a superposition of the scattering processes of the so-called "virtual" photons.

Weight parameter can be described by some functional. In this case its maximum value can be estimated from the minimum condition, which allows you to build weight windows with a minimum dispersion. Then, when the initial photon weight is $\varpi$, as a result of a reflection from the boundary of the separation of media with different refractive indices it will change: $\varpi \cdot R$, but for the photon that passed into the medium - $\varpi(1-R)$. If some falling photon gain the weight $-\varpi(1-R)$, then for the reflection of the boundary of division weight will equals $R$, and for the photone which has passed through it $-\varpi(1-R)$. Change of photons weight throught scattering and absorption is determines as $\varpi \frac{\mu_{\alpha}}{\mu_{\alpha}+\mu_{S}}$. So, after $n$ scattering acts of photons in the medium, their flows at a distance of $z=\frac{n}{\mu_{\alpha}+\mu_{S}}$ equals $\varpi\left(1-\frac{\mu_{\alpha}}{\mu_{\alpha}+\mu_{S}}\right)^{n}==\varpi \exp \left(-n \frac{\mu_{\alpha}}{\mu_{\alpha}+\mu_{S}}\right)$. Thus, if the distance between two consecutive scattering acts of a photon is chosen from the logarithmic distribution generated by the computer, then, in order to take account of the act of absorption, each photon is assigned a weight $\varpi$, which decreases continuously during the propagation of a photon in the absorbing medium. At the entrance to the medium $\varpi=1$.

The algorithm for modeling the interactions of photons with the medium by the MCM is based on the following basic steps: photon source generation, trajectory generation, absorption, destruction, photon registration. Sometimes so-called "ballistic photons" are introduced, which, without losing their weight, come out of a scattering medium. The process of diffuse scattering is also conveniently divided into several types. Because, a photon can undergo diffuse scattering and be transferred to the outflow from the medium 
along the trajectory in the form of a "snake" and, in general, can not escape with the only diffuse scattering. Each elementary act of interaction of a photon with a medium is characterized by a change of its weight $\Delta \varpi=\left(1-\frac{\mu_{S}}{\mu_{\alpha}+\mu_{S}}\right)$.

Algorithmization of modeling of a random photon trajectory in a scattering medium. The photon source is located at the origin $(0,0,0)$ on a three-dimensional coordinate grid (Fig.1d). Let's set the algorithm of the drawing of the parameter, which determines the direction of propagation of the scattered photon. Since the scattering diagram is symmetric according to the direction of the fall of the photon ( $\mathrm{Z}$ axis), this statistical symmetry in the statistical simulation of the probability of the azimuthal scattering direction is equivalent to the requirement that the cosine of this angle be uniformly distributed in the range $[-1 ;+1]$, thus, the random choice of the scattering direction is modeled by the equation $\cos \theta=2 r-1$.

Let's consider the scheme of calculation of the random variable describing the parameters of the random trajectory of the photon motion in the medium. Let'i the photon gained $k$-scattering at the coordinate point $z_{k}$ and after scattering began to move in the direction $\cos \theta_{k}$. Let`s play the mean free path with formula $\lambda_{k}=-\frac{1}{\sigma} \ln r$ and calculate the abscissa (coordinate) of the next scattering) $z_{k+1}=z_{k}+\lambda_{k} \cos \theta_{k}=z_{k}+\lambda_{k}(2 r-1)$.Let's check the condition of passing a photon through a layer: $z_{k+1}>\mathrm{h}$. If this condition is not satisfied, the photon count is completed and a unit is added to the photon counter passing through the layer. Otherwise check the condition $z_{k+1}<0$. If this condition is not met, then the calculation of the photon trajectory is completed and a unit is added to the counter reflected by a layer of photons. If this condition is not fulfilled as well, i.e. $0 \leq z_{k+1} \leq h$, then this means that the photon has undergone another collision inside the layer and it is necessary to play the part of the photon in a collision.

In this case, new value $r$, should be taken and photon absorption condition $r<\frac{\mu_{\alpha}}{\mu}$ should be checked. If the last inequality is satisfied, then the trajectory calculation is stopped and a unit is added to the counter of the absorbed photons. Otherwise, we assume that the photon was scattered at the point with the abscissa $z_{k+1}$. Then a new direction of motion of the photon is played $\cos \theta_{k+1}=2 r-1$ and the whole cycle is repeated again, each time with the new values of the variable $r$. All $r$ are recorded without indexes, since it is assumed that each collision is used only once. Three values of $r$ are needed to calculate the one section of photons path. Initial values for each photon path: $z_{0}=0, \cos \theta_{0}=1$.

Let the incident light beam consists of a certain number of photons entering the medium plane $(X, Y)$ along the axis $Z$ at a coordinate point $(0,0,0)$. If the photon is swallowed, then a new one is launched. The calculation continues until the photon does not absorb or will not go beyond the medium. The value of the angle of the total internal reflection $\left(R\left(\theta_{\kappa p}\right) \rightarrow 1\right)$ is calculated by the formula $\theta_{\kappa p}=\sin ^{-1}\left(\frac{1}{n_{\text {onm }}}\right)$. With a total internal reflection of a photon, a random number is generated $r \in[0 ; 1]$, which is compared with the value of the energy reflection coefficient. In case of $r \leq R\left(\theta_{\kappa p}\right)$ the photon undergoes the total internal reflection, otherwise it is assumed that it leaves the medium. For the photon that has undergone the total internal reflection, the value of the coordinate inverts $z \leftarrow-z$, and other coordinates remain unchanged. For the angles of incidence exceeding this critical value, the energy reflection coefficient equals $R(\theta)=\frac{1}{2}\left[\frac{\sin ^{2}\left(\theta_{\kappa p}-\theta\right)}{\sin ^{2}\left(\theta_{\kappa p}+\theta\right)}+\frac{\operatorname{tg}^{2}\left(\theta_{\kappa p}-\theta\right)}{\operatorname{tg}^{2}\left(\theta_{\kappa p}+\theta\right)}\right]$. 
01

12

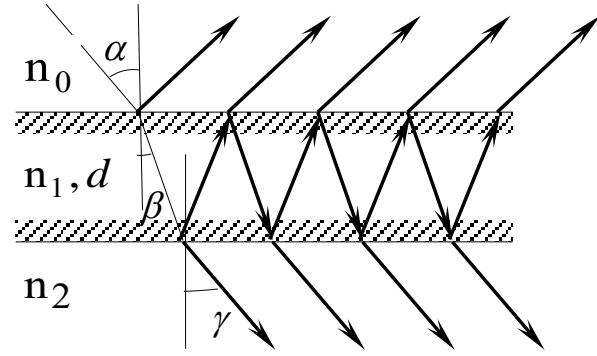

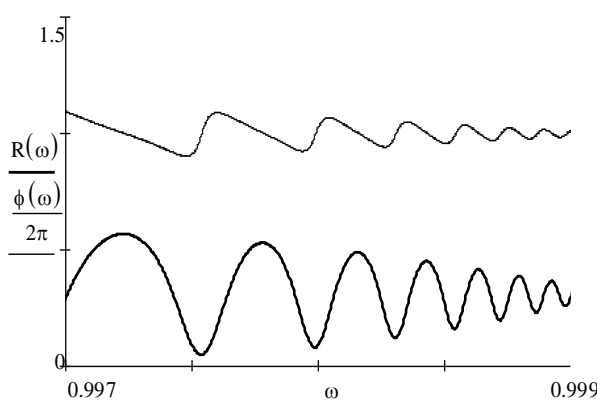

b

Fig.4

Reflections coefficient $\tilde{n}_{2}$ of plane-parallel plate with thickness $d$ and refrective index $\tilde{n}_{2}$ is calculated with formula $R_{s p}=\operatorname{Re}^{2} \tilde{r}_{s p}+\operatorname{Im}^{2} r_{s p}$, where Fabri-Perot multi-beam interference effect is taken into account with an representation of amplitude reflection coefficient as $\tilde{r}_{s p}=\frac{\tilde{r}_{12}+\tilde{r}_{23} \exp (-i \tilde{\delta})}{1+\tilde{r}_{12} \tilde{r}_{23} \exp (-i \tilde{\delta})}$, $\tilde{\delta}_{j}=\frac{4 \pi n_{2} d}{\lambda} \cos \theta_{j+1}$ simultaneously with a low law of refraction $\tilde{n}_{j-1} \sin \theta_{j-1}=\tilde{n}_{j} \sin \theta_{j}=\tilde{n}_{j+1} \sin \theta_{j+1}, d$

- distance between two plane-parallel fronts, formed by indexes $j-1, j, j+1$. The scheme of superposition amplitudes of Fabri-Perot is depicted in Fig. 4.a. Modulation of reflection coefficient $R(\omega)$ and phase $\phi(\omega)$ of light, reflected by plane-parallel plate is shown in Fig. 4b, where the relative permittivity gains dispersion. Calculations are performed according to the formulas: $R=\frac{R_{m}+b^{2} \cos ^{2} F_{-}}{1+b^{2} \cos ^{2} F_{+}}=\frac{R_{M}-a^{2} \sin ^{2} F_{-}}{1-a^{2} \sin ^{2} F_{+}}$, $R_{M, m}=\left(\frac{\sigma_{01} \pm \Theta}{1 \pm \sigma_{01} \Theta}\right)^{2}, \quad a^{2}=\frac{4 \sigma_{01} \Theta}{\left(1+\sigma_{01} \Theta\right)^{2}}, b^{2}=\frac{4 \sigma_{01} \Theta}{\left(1-\sigma_{01} \Theta\right)^{2}}, F_{ \pm}=\frac{1}{2}\left(\phi_{01} \pm \xi\right), \xi=\phi_{12}-\operatorname{Re} \tilde{\delta}, \Theta=\Omega \sigma_{12}$, $\Omega=\exp (-\operatorname{Im} \tilde{\delta})$, indices $1,2,3$ correspond to the numbers of mediums, respectively, above the plate (1) and under it -3 (Figure 4 a)[12]

Consider the 3D-process of elastic scattering photons in an optically inhomogeneous medium of a cubic shape with the length of the side $a: a:=1000 ; \min X:=-0.5 a ; \max X:=0.5 a ; \min Y:=-0.5 a$ $; \max Y:=0.5 a ; \min Z:=-0.5 a ; \max Z:=0.5 a$. Then the function of the photon presence in it is going to be: ${ }^{a}(\mathrm{x}, \mathrm{y}, \mathrm{z}):=(\min \mathrm{X}<\mathrm{x}<\operatorname{maxX}) \cdot(\min \mathrm{Y}<\mathrm{y}<\max \mathrm{Y}) \cdot(\min \mathrm{Z}<\mathrm{z}<\max \mathrm{Z})$. This function returns a value of 1 for the coordinate values inside the cube and 0 outside it: $a(0,0,0)=1 \quad a(500,0,0)=0$, etc. For $a$ constant value of the scattering parameter $\mu s$, the intensity of light decreases according to the exponential law, therefore the random value of the mean free path will belong to the distribution $-\log (r n d(1)) / \mu s$. The Monte Carlo algorithm for scattering of the photon has the form (1).

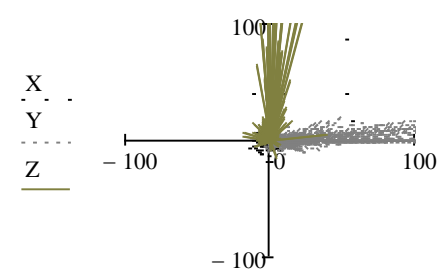

Y, Z, X

a) $g=0$

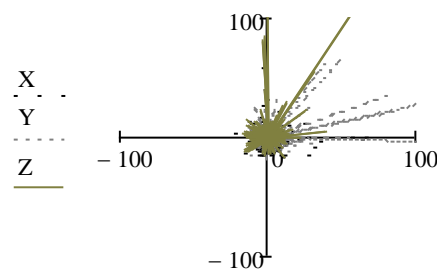

Y, Z, X

b) $\mathrm{g} \neq 0$

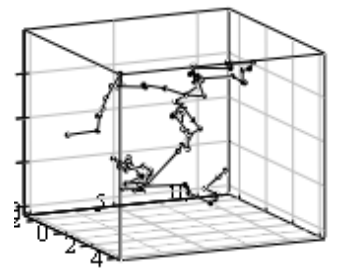

(X, Y,Z)

c)

Fig. 5 
Fig.5 a,b shows the flat sections of 3D scattering diagrams of photons reflecting the anisotropy effect. Fig. 5c and d show the trajectories of diffuse motion: on the left for one photon, which carried out 100 acts of scattering, and on the right - for 10 photons with the same number of acts of scattering. In Fig. $5 \mathrm{c}$, the visualization of random sequences returned by the function $\operatorname{Walk}()$ and and their histograms are given. We are convinced of the equally probable distribution of the coordinates of the scattered photon. Authors advice is taken into consideration in the algorithm [13].

Figure 4 also shows the curve of theoretical distributions as histogram envelopes. Based on the criterion of "chi-square" we justified the distributions of relative frequency values $R$ with $95 \%$ confidence probability will be normal in both cases. On these figures also plotted confidence limits with confidence probability of $95 \%$ for the reflection coefficient $R$ that determine confidence interval.
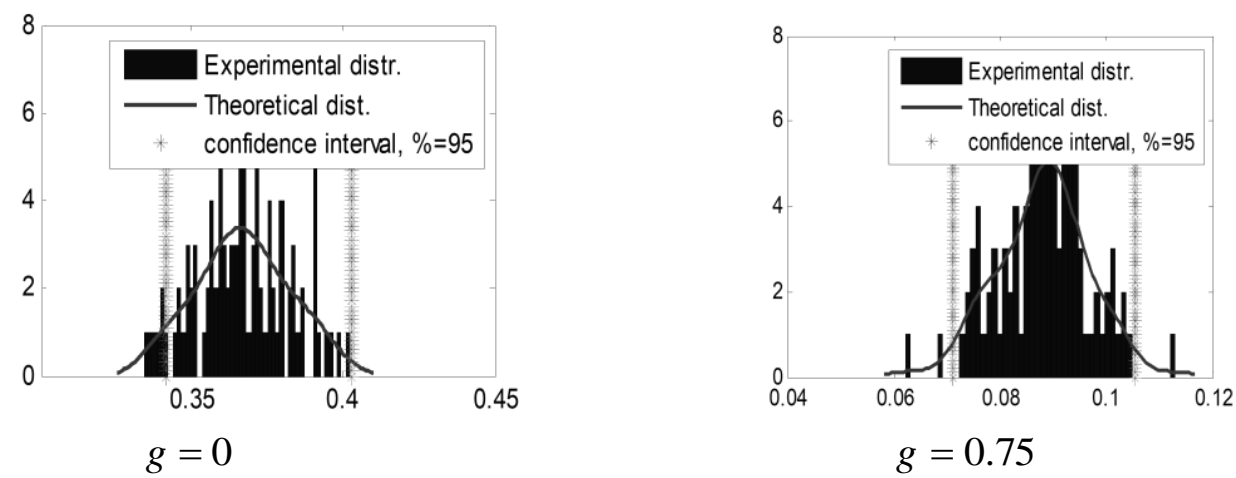

Fig. 6. Distribution of the relative frequency values of the diffuse reflectance ratio

Taking into account in the integral law the intensity attenuation of light by plane-parallel layer of spatial nonlinearity transport coefficient of photons interaction with scattering centers does not significantly change the width of the confidence interval for the same confidence probability. Here the value of other parameters $\mu_{s}=90 \mathrm{~cm}^{-1}, \mu_{a}=10 \mathrm{~cm}^{-1}$ and thickness of the plane parallel layer $\max Z=0.02 \mathrm{~cm}$ are taken similar to [14]. We see that taking into account the appropriate nonlinearity leads to some increase in the average values of the diffuse reflection $m e a n R$. And the difference meanR $\left.\right|_{g \neq 0}-$ meanR $\left.\right|_{g=0}$ for diverse values of the anisotropy $g$ does not significantly change, coefficient of photons interaction.

\section{Conclusion}

The Monte Carlo method proposed by Stanislav Ulam is effective for modeling random processes in optical systems with fluctuations, the physical expression of which is the value of the variance. In the three-dimensional random scattering model, the minimum set of statistically independent random values of the parameters consists of the polar and zenith angles and the free path length.

\section{Reference}

1. Kosobutskyy P. Statistical and Monte-Carlo Algoritms for Simulation of Random Processes in macro- and microsystems using MathCAD. Lviv: LPNU, 2014;

2.Wang L., Jacques S. Monte-Carlo modeling of light transport in Multi-layer Tissues in Standart C. University of Texas. Anderson Cancer Center, 1992;

3.Wang L., Jacques S.,Zheng L. MCML-Monte-Carlo modeling of light transport in multi-layer tissues. Computer Methods and Programs in Biomedicine.-Vol.47.-P.131-146 (1995).

4.Binzoni T., Leung T.S., Gandjbakhche et.al. The use of the Heyey-Greenstein phase function in Monte Carlo simuliations in biomedical optics. Phys. Med.Biol.Vol.51.-P.N313-N322.-2006.

5. Prahl S.A., Light transport in tissue, PhD dissertation, University of Texas at Austin, 1988.

6. Wang L.H., Jacques S.L., Monte Carlo Modeling of Light Transport in Multi-layered Tissues in Standard C, University of Texas, M. D. Anderson Cancer Center, 1992. 
7.Wang L.H., Jacques S.L., Zheng L.-Q., MCML-Monte Carlo modeling of photon transport in multi-layered tissues, Computer Methods and Programs in Biomedicine, vol. 47, pp. 131-146, 1995.

8.Zhu C., Liu Q., Review of Monte Carlo modeling of light transport in tissues, Journal of Biomedical Optics 18(5), 050902, May 2013.

9. Jacques S. L., Optical properties of biological tissues: a review, Phys. Med. Biol. 58, 2013, pp.3761.17. Vo-Dinh T., Biomedical Photonics handbook, CRC Press, March 2003.

10. Wang L.V., Wu H.I., Biomedical Optics: Principles and Imaging, Wiley, 2007.

11. Boas D.A., Pitris C., Ramanujam N., Handbook of Biomedical Optics. CRC Press June 2011.

12. Kosobutskyy P. Envelope Method in Fabry-Perot Interferometry. Lviv: LPNU, 2013.

13. PH36010 Numerical Methods. Writing Programs in MathCAD.

14. Salerud E.G., Biomedical Optics: Light scattering in Tissue by Monte Carlo Simulation, IMT October 2006.

П. Кособуцький, М. Лобур, Ю. Матвіїв-Лозинська

Національний університет «Львівська політехніка»

\section{МЕТОД СТАТИСТИЧНОЇ ІМІТАЦЇ,, С. УЛАМА ТА ОСНОВНІ ПРИНЦИПИ ЗАСТОСУВАННЯ ДЛЯ МОДЕЛЮВАННЯ ВИПАДКОВНИХ ПРОЦЕСІВ}

(ㄷ) Кособуцьький П., Лобур М., Матвї̈в-Лозинська Ю., 2021

Стаття присвячена відомим львів'янам, автору методу Монте-Карло, та математику Марку Кацу. В статті коротко розкриті методичні алгоритми Монте-Карло моделювання статистичних закономірностей проходження світла крізь оптично неоднорідні середовища.

Ключові слова: метод Монте-Карло, розсіяння світла мутними середовищами 